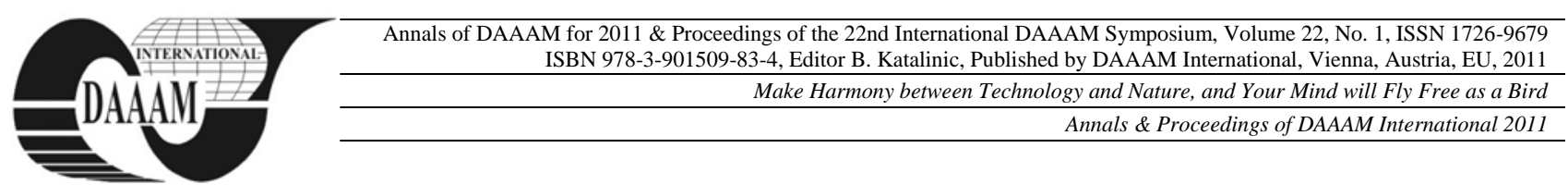

\title{
CONTRIBUTION SPECTROMECTRICAL ANALYSIS OF THERMAL PLASMA
}

\author{
GUTT, S[onia]; GUTT, G[heorghe]; ALEXUC, F[lorin] - C[ristian]; TODIRICA, F[lorin] S[orin]; \\ GUTT, A[ndrei] \& SEVERIN, T[raian] - L[ucian]
}

\begin{abstract}
The equipment described in the paper consists of a portable optoelectronic structure meant to determine in situ the element chemical composition, metallic material of a welding seam, basic metallic material welded, metallic walls of plasma or gas cutting joint, material welded or cut by laser, some charges of melted steel as well as some other high energy processes accompanied by emission of light electromagnetic radiation, using exactly the high energy radiation in the spectrometer analysis of these heat sources. The device structure and its technical feasibilities are described, too

Key words: emission, spectrometry, laser telemetry, video analysis
\end{abstract}

\section{INTRODUCTION}

The devices used in the spectrometry analysis of emission use different heat sources such as flame, spark or electric arc, plasma and laser to excite the matter analyzed.( Zarval'ska, et al.2000), (Gianchandani., 2009). Most often the price of these spectral sources is higher than that of optoelectronics spectral analysis itself. Also, the presence of these sources, their dimension as well as the need to get them supplied from network, turn the spectrometers of spectral analysis of emission into fixed laboratory instruments (Agresti,2009), excepting stylometers which are portable spark-arc spectrometers, instruments that require however current supply from a relatively high electric source to produce the spark or electric arc. The use of thermal plasma from different processes as spectral source (Gutt et al 2010-1, 2010-2, 2010-3) eliminates all these disadvantages.

\section{EXPERIMENTAL}

For the qualitative and quantitative spectral analysis of some materials brought to incandescence by technological methods, natural phenomena, incidents or accidents, the authors have thought, designed and made an unit portable optoelectronic structure supplied by electric energy by means of the USB source of portable computer, consisting of three optical channels as follows:

- an optical channel which comprises a miniature fixed diffraction-network and Diode Array detector and USB interface spectrometer connected to portable computer meant to chemical element qualitative and quantitative analysis of the material brought to plasma state by a heat process of the above mentioned ones

- an optical channel which comprises a video camera connected to the portable computer - meant to on line and in situ following of the heat process area and optical axis centring quality of spectrometer on the maximum spectral emission

- an optical channel which comprises a digital laser telemeter connected to the portable computer- meant to measure and fix the required distance between radiation source and video spectrometer as well as to centre the optical axis of spectrometer on the source of spectral radiation using the targeting of maximum emission area with red beam of laser radiation

- a semitransparent dichroic plane mirror for eyes' protection against ultraviolet radiation of spectral source, which permits the targeting beam to be followed by laser

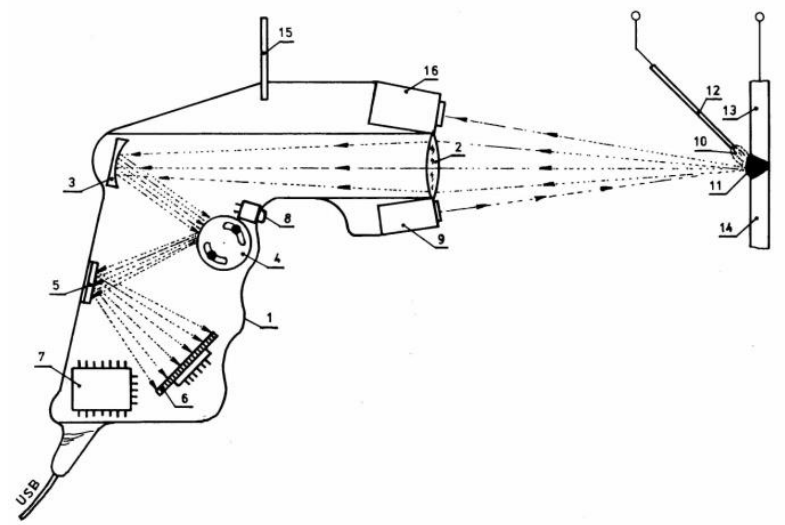

Fig. 1. Overview of portable optoelectronic structure of the device during application regarding the qualitative and semi quantitative analysis of welding seam at electric welding. 1body, 2-collimating lens, 3-optical plane mirror, 4-diffraction network, 5-optical mirror, 6-Diode-Array detector, 7-interface of USB type, 8-starter, 9-laser telemeter, 10-electric arc, 11welding seam, 12-tip electrode , 13,14-basic material, 15-semi transparent mirror, 16- video camera

From the constructive point of view, the spectrometer consists of a body 1, figure 1, inside which a miniature spectrometer is placed, which at its turn is comprised by a collecting lens 2 , an optical collimating lens 3 , a diffraction network 4 , an optical mirror of total reflection 5, a Diode-Array detector 6, an USB type interface 7 , a laser telemeter 9 , an optical channel made by a video camera 16. Qualitative and quantitative element chemical composition of a welding seam 10 , made by an electric arc 11 between a tip electrode 12 and the basic material 13,14 or qualitative and quantitative element chemical composition of any other heat plasma sources is automatically determined by means of a portable computer which provides, together with a specialized programme, the spectral and composition data acquisition, processing and display, video image of the area focused on as well as telemeter and optical centring data of spectrometer as against the radiation source.

\section{RESULTS AND DISCUSSION}

Given that for making semi quantitative analysis under as accurate as possible conditions it is necessary for the emission radiation intensity to be always measured under the same conditions, at plasma's maximum emission intensity respectively. With this aim in view it is necessary for the optical axis of collimating collecting lens to fall on the area of maximum emission intensity, condition provided by the laser 


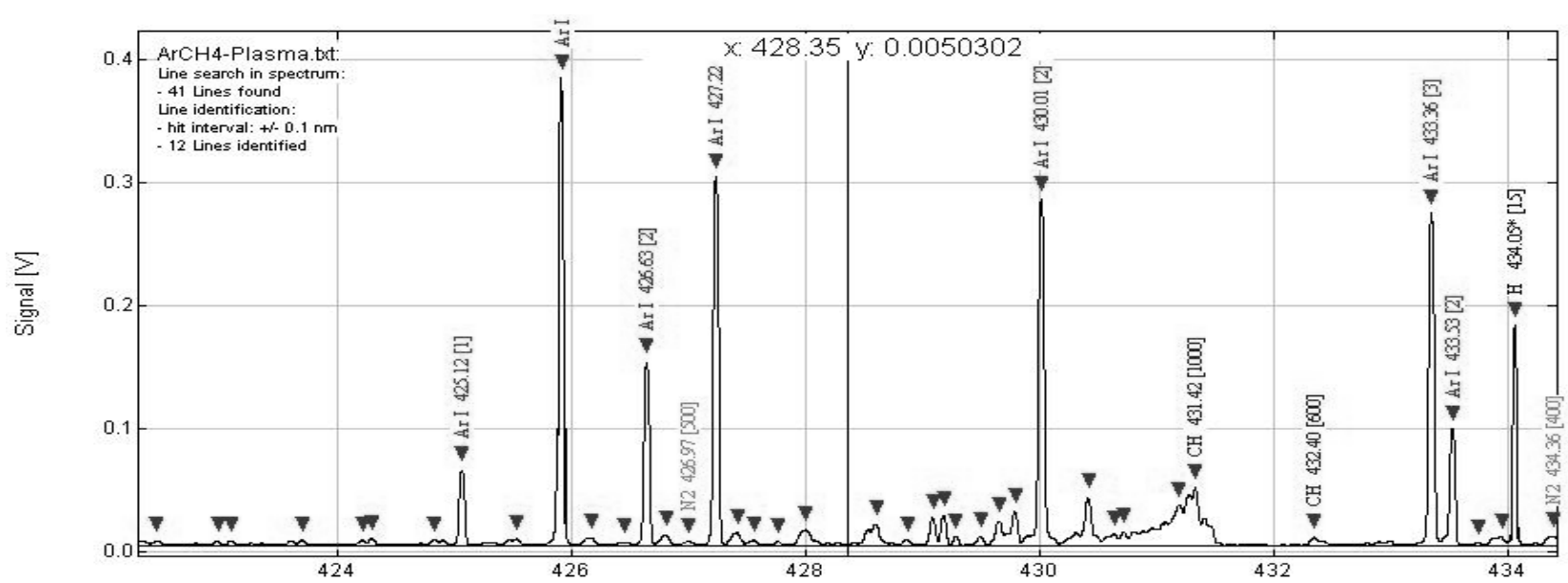

Fig. 2. Welding seam spectrogram, in protecting medium of argon, made by portable spectrometer and spectral SpecLine programme of company OceanOptics USA

targeting system, and while the spectrum is being acquired, the spectral source should be found at the focal point of collecting lens. To meet the last requirement, it is necessary both to know continuously the distance between lens and seam and keep this distance constant during spectrum acquisition, which is very difficult to be achieved as the spectrometer is hand held.

Solving this problem out was possible due to the suggestions made by the authors in conceiving and carrying out the spectrometer hereby shown. According to these solutions, the control of spectra acquisition is made only for maximum emission intensities and for very short periods of time of milliseconds, in the end a single spectrum, for a measuring point, results which is the spectral average of tens of spectra acquired. Since maximum emission intensities give maximum photocurrents at Diode Array detector, to sense these ones and control spectrum acquisition, we used the moment when the result of I derivate of photocurrents' sum (determined by the integration of photocurrents' sum $\sum I_{f}$ given by Diode- Array detector and time $t$ has the value zero:

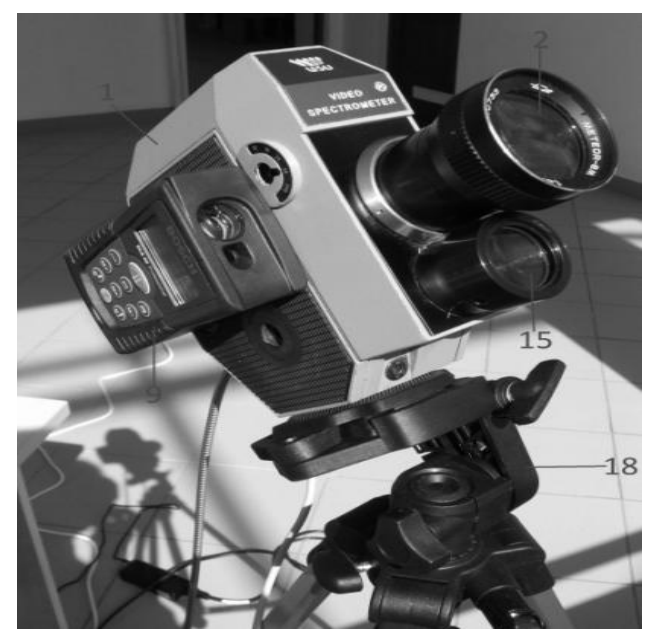

Fig. 3. View of emission video spectrometer made at the University Suceava. 1-body of video spectrometer, 2collimating lens, 9-laser telemeter, 15-video camera, 18-tripod

$$
\frac{d \sum I_{f}}{d t}=0
$$

Derivation is electronically made at intervals of milliseconds, and acquisition time of spectrum is determined by modern spectrometers of the type used in achieving the instrument in question, in the same field of milliseconds. The spectrometer in figure 3 is based on the concept presented, made by own means and is under the form of functional prototype at the University "Stefan cel Mare" Suceava. Figure 2 shows the spectrogram of a welding seam, in protecting medium of argon, made by the portable spectrometer described and spectral programme SpecLine of the company OceanOptics USA.

\section{CONCLUSIONS}

Using the emission radiation of some heat processes of high intensity as spectral source we made a portable and compact video spectrometer, meant to analyze in situ the composition of welding metallic seams, basic material, heat-cut metallic materials, charges of melted metal, explosions, burning processes, volcanoes' lava. The use of video spectrometer permits to increase accuracy of determining the component concentration of certain material as the final spectrum, which the semi quantitative analysis relies on, is the average result of more spectra acquired successively at the moment of reaching the conditions of maximum spectral emission. The use of laser telemeter leads also to the increase of accuracy which permits continuous measurement and maintenance of the distance between spectral source of emission and spectrometer, with the same telemeter being possible to centre the optical axis of spectrometer on the maximum spectral emission zone.

\section{REFERENCES}

Agresti J., A. A. Mencaglia and S. Siano (2009) Development and application of a portable LIPS system for characterising copper alloy artefacts, Analytical and Bioanalytical Chemistry, Volume 395, Number 7, p. 2255-2262

Gianchandani Y. B., Wright S. A., Eun C. K., Wilson C. G., Mitra B., (2009) Exploring microdischarges for portable sensing applications, Rewiew, Analytical and Bioanalytical Chemistry Volume 395, Number 3, p.559-575

Gutt G., Gutt S., Todirică F.S., (2010-1), Emission portable spectrometer, A00874, Patent proposals, (romanian), OSIM Bucuresti

Gutt G., Gutt S., Todirică F.S., (2010-2), Video Spectrometer, A00872, Patent proposals, (romanian), OSIM Bucuresti

Gutt G., Gutt S., Poroch-Seritan M., Gutt A., (2010-3), Spectrometric Device , A00871, Patent proposals, (romanian), OSIM Bucuresti

Zarval'ska A., Ovsik Ya., and Ulanovskii M., V., (2000), Portable laser microanalyzer, Measurement Techniques, Volume 43, Number 7, p.602-606 